\title{
Electrochemical determination of erythromycin in drinking water resources by screen printed carbon electrodes
}

\author{
Albana Veseli ${ }^{1}$, Ferdane Mullallari ${ }^{1}$, Festina Balidemaj ${ }^{2}$, L'ubomír Švorc ${ }^{3}$, Tahir Arbneshi ${ }^{1}$ \\ ${ }^{1}$ Department of Chemistry, Faculty of Natural and Mathematical Science, University of Prishtina \\ 'Hasan Prishtina' Nëna Terezë, 10000 Prishtina, Republic of Kosovo \\ ${ }^{2}$ Rochester Institute of Technology, Prishtina \\ ${ }^{3}$ Institute of Analytical Chemistry, Faculty of Chemical and Food Technology, Slovak University of \\ Technology in Bratislava, Radlinského 9, Bratislava SK-812 37, Slovak Republic \\ albana.veseli@uni-pr.edu
}

\begin{abstract}
:
Erythromycin (ERY) belongs to a class of antibiotics that is suggested as one of the prior drinking water contaminants at latest European Union Water Framework Directive (EU - WDF). Therefore, the main goal of this study was to develop a new and sensitive analytical method for quantitative determination of ERY based on the use of screen printed carbon electrodes (SPCEs) modified with sodium dodecyl sulfate. The electrochemical oxidation of ERY was investigated for the purpose of its flow-injection amperometric determination (in phosphate buffer solution $\mathrm{pH} 8.50$ ) with a detection potential of $-700 \mathrm{mV}$. Using flow injection analysis (FIA) the modified SPCE showed a linear dynamic range up to $15 \mathrm{mg} / \mathrm{L}$ with a detection limit $(3 \sigma)$ of $0.14 \mathrm{mg} / \mathrm{L}\left(E_{d}=-700 \mathrm{mV}\right)$. The reproducibility of the amperometric detection with the five different SPCEs was expressed as RSD with the achieved value of $2.99 \%$ for five successive injections of $5 \mathrm{mg} / \mathrm{L}$ of ERY and did not present any memory effect. The proposed sensor is low-cost and easily applicable by user in real samples. Optimized method was applied for the determination of ERY in pretreated surface water. Finally, the applicability of using SPCEs for the electrochemical detection of ERY (i.e. for quality control analysis) was demonstrated by using FIA in a surface pretreated water spiked samples.
\end{abstract}

Key words: erythromycin, sodium dodecyl sulphate, flow injection analysis, amperometric detection

\section{Introduction}

Erythromycin (ERY) is a broad - spectrum macrolide antibiotic that contains 14 membered lactone rings with 10 asymmetric centers and 2 sugar molecules which can be formed by Streptomyces erythraesus during the fermentation process. Over the past decades the use of antibiotics has increased dramatically and consequently the existence of antibiotics in aquatic environment has been detected contaminating drinking waters and lead to some serious adverse effects on human health as well as on wildlife. When the drinking waters are exposed to antibiotics, bacteria gain resistance against them leading in one of the very actual healthy problem, bacteria resistance.

Based on the literature [1-3] electrochemical methods have been proved to be excellent alternatives in the analytical chemistry field due to their simplicity, low cost, short analysis times and high sensitivity.

\section{Materials and methods}

All used reagents were of analytical reagent grade and used directly without purification. Cyclic voltammetry was applied using batch voltammetric system consisted of a potentiostat (PalmSens, The Netherlands) connected to a laptop computer using the corresponding software for data analysis (PSTrace).

Flow injection experiments were performed using a thin-layer electrochemical cell (CC-5, BASi), a peristaltic pump (Pharmacia Fine Chemicals, Sweden), a sample injection valve (5020 Rheodyne, Cotati, CA, USA), and an electrochemical workstation (PSTrace). end of the SPCE, to which a crocodile clamp was attached for electrical contact. Screen printed carbon electrodes (SPCEs) were made using carbon ink (Electrodag $421 \mathrm{SS}$, Acheson) as a material for printing. Before application of SPCE, $20 \mu \mathrm{L}$ of the $0.01 \mathrm{M}$ solution of sodium 
dodecyl sulphate (SDS) were dropped on the electrode surface. The printed electrodes were dried at room temperature for $15 \mathrm{~min}$ and then were cut into individual sensor strips.

\section{Results and discussions}

Cyclic voltammograms were recorded (Fig. 1) with modified SPCEs from $0.5 \mathrm{~V}$ to $1.2 \mathrm{~V}$ to find the basic electrochemical information for the analyte. Based on the results the oxidation of ERY started from $0.7 \mathrm{~V}$. The reproducibility of the signal proved the stability and the effectivity of the sensor for ERY determination. Flow injection analyses were carried out using an operation potential of $700 \mathrm{mV}$ (or alternatively $750 \mathrm{mV}$ ). The responses were evaluated using the peak height (Fig. 2).

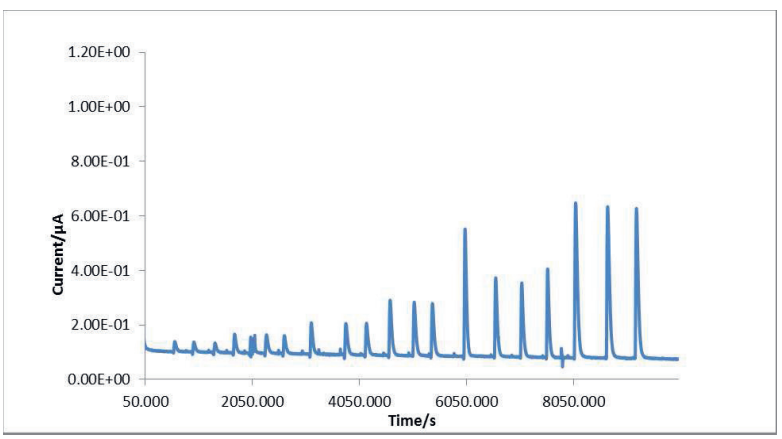

Fig.2. The typical FIA record for ERY determination; carrier phosphate buffer (0.1 M pH 8.5), operation potential of $700 \mathrm{mV}$, flow rate of $0.4 \mathrm{~mL} / \mathrm{min}$, injection volume of $200 \mu \mathrm{L}$.

\section{Conclusion}

In this study, sensor based on SPCEs modified by SDS was developed for selective and sensitive determination of ERY in water samples. The physical adsorption of the surface SDS modifier improved the performance of the sensor and consequently has also improved the selectivity and the sensitivity of the method. An LOD of $0.14 \mathrm{mg} / \mathrm{L}$ could be achieved with a dynamic range up to $15 \mathrm{mg} / \mathrm{L}$; the method was applied successfully to the determination of ERY in spiked water samples using standard addition method.

\section{Acknowledgments}

We are grateful to the Ministry of Education, Science and Technology for financial support (Project No. 2-5521-3). L'Š wishes to acknowledge the Grant Agency of the Slovak Republic (Grant No. 1/0489/16).

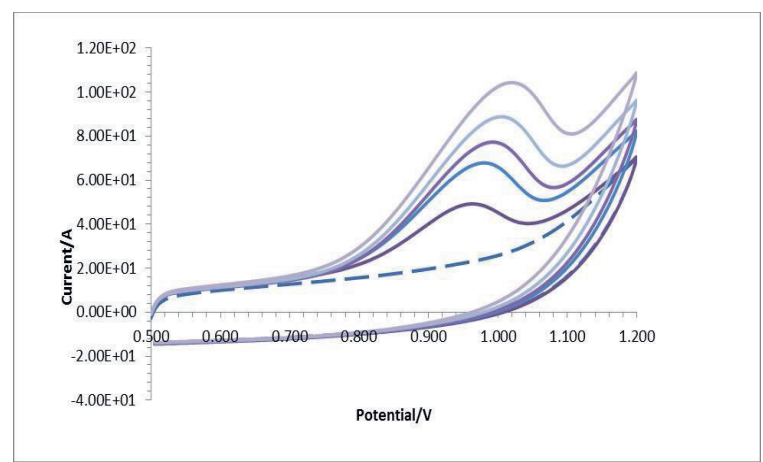

Fig.1. Cyclic voltammograms of ERY at a SPCES modified with SDS; carrier phosphate buffer solution of $0.1 \mathrm{M}, \mathrm{pH}$ 8.5; scan rate of $100 \mathrm{mV} / \mathrm{s}$; solid curve blank, dashed curve - blank, solid curves with successive addition of $20 \mathrm{mg} / \mathrm{L}$ ERY.

\section{Practical application in real samples}

Using standard addition method the determination of ERY was performed in two different samples of pretreated water (A, B), which were taken from two water public factories for drinking in the Prishtina region. The results are shown in Table 1.

Tab. 1: Determination of ERY in two surface water samples

\begin{tabular}{|c|c|c|}
\hline Sample & $\begin{array}{c}\text { Found } \\
\text { (mg/L) }\end{array}$ & RSD (\%) \\
\hline A & $8.5 \times 10^{-2}$ & 1.7 \\
\hline $\mathrm{B}$ & $7.1 \times 10^{-3}$ & 6.9 \\
\hline
\end{tabular}

\section{References}

[1] M. Okan, M. Duman, Functional polymeric nanoparticle decorated microcantilever sensor for specific detection of erythromycin, Sensors and Actuators B 256, 325 - 333 (2018); doi:10.1016/j.snb.2017.10.098

[2] X. Hu, P. Wang, J. Yang, B. Zhang, J. Li, J. Luo, $\mathrm{K}$. Wu, Enhanced electrochemical detection of erythromycin based on acetylene black nanoparticles, Colloids and Surfaces B: Biointerfaces 81, 27 - 31 (2010); doi:10.1016/j.colsurfb.2010.06.018

[3] M. Egervärn, S. Englund, M. Ljunge, CH. Wiberg, M. Finn, M. Lindblad, S. Börjesson, Unexpected common occurrence of tansferable extended spectrum cephalosporinase - producing Escherchia coli in Swedish surface waters used for drinking water supply, Science of the Total Environment 587 - 588, 466 - 472 (2017); doi: 10.1016/j.scitotenv.2017.02.157 